\title{
The International EQUATOR Network: enhancing the quality and transparency of health care research
}

\author{
Nikolaos PANDIS ${ }^{1}$, Zbys FEDOROWICZ² \\ 1- DDS, MS Dr. med. dent. MSc, Private Practice, Corfu, Greece, Visiting Assistant Professor, Department of Orthodontics \\ and Dentofacial Orthopedics, Dental School / Medical Faculty, University of Bern, Switzerland
}

2- MSc DPH, BDS LDS RCS Eng., Director, The Bahrain Branch UK Cochrane Centre, Bahrain

As the number of scientific publications in the biomedical field increases exponentially and as it has become apparent that appropriate synthesis of individual study results is critical in clinical decision making, there is a need for improved reporting of the published studies. One of the first and most comprehensive attempts towards improved reporting was the publication, in 1996, of the core CONSORT guidelines (Consolidated Standards for Reporting Trials) by the CONSORT group. The first CONSORT document was also followed by the CONSORT extension guidelines covering trials with specific designs such as cluster randomized and non-inferiority trials (http://www.consort-statement.org/aboutconsort). The third and latest version of the core CONSORT document was published in the spring of 2010.

A further initiative towards better reporting and a development from the CONSORT group is the EQUATOR network (Enhancing the Quality and Transparency of Health Research) (http: // www.equator-network. org/) . As the EQUATOR group states:" The EQUATOR Network is an 'umbrella' organization that brings together researchers, medical journal editors, peer reviewers, developers of reporting guidelines, research funding bodies and other collaborators with mutual interest in improving the quality of research publications and of research itself."

Currently the EQUATOR network site has a plethora of published guidelines and other relevant publications to assist in research reporting all "interested" in a one stop shop package. A reasonable question that would come to mind is the following: how is the EQUATOR network initiative for improved reporting in healthcare can help dental research and dentistry in general? The answer to this question is that dentistry is bound to benefit at several levels.

Accurate and transparent reporting facilitates correct indexing, easier retrieval, assessment and utilization of the published reports by the investigators seeking to evaluate the available evidence on the topic of interest. Although the guidelines in the EQUATOR pertain mainly to reporting rather than design and quality assessment of health related research, there are potential positive implications for an improved study design. Prospective researchers planning with the end in mind may use the guidelines at the design stage to make sure that all important aspects of the research project are being considered. Therefore, a group of investigators about to design a randomized clinical trial or an observational study may refer to the CONSORT or the STROBE guidelines respectively and use them as checklists to make sure important design issues are considered and accounted for at the initial stages of the project.

The editorial process is a demanding and very responsible task as the editors must act as the "gatekeepers" to the knowledge. It is important for the editors to be able to separate "good" from "bad" research as this may have important consequences on clinical practice and patient well-being. Healthcare authorities rely on published research for preparing healthcare policies and guidelines, and the clinicians may base their decision making process on those drafted guidelines. Therefore, it is easy to understand that accurate and transparent reporting of what was actually done during the research study is vital in assessing the worthiness of the research and whether it deserves to be seen by the public eye. Editors may adopt pertinent guidelines from the EQUATOR initiative in order to promote better reporting in the journals, and In fact, a large number of journals has already adopted the CONSORT and other relevant guidelines.

The peer reviewing process goes hand in hand with the editor's work, and may be greatly enhanced as the reporting guidelines can be used as a reference checklist of what should be included in the submitted manuscript. Adherence to good reporting by the authors improves the whole peer reviewing process for the benefit of editorial boards, authors and patients. Unfortunately, although a large number of journals have adopted guidelines such as the CONSORT, adherence to those guidelines appears to have been suboptimal because it seems that there is a lack of their enforcement by the editorial group to the authors. This is an area of great concern to the EQUATOR network and monitoring improvement of adherence to the guidelines is one of the key objectives of the group's five year plan. It is of interest to note that as of July 2011, the American Journal of Orthodontics and Dentofacial Orthopedics has gone a step further toward better reporting by assuring, via an internal process and before peer-reviewing commences, adherence of randomized clinical trials and systematic reviews to the CONSORT and PRISMA guidelines, respectively. http://journals. elsevierhealth.com/periodicals/ymod/authorinfo.

The investigator seeking information to enhance his current or future research project or who is interested in conducting a systematic review in order to get a more precise answer to a question of clinical importance will also reap the benefits. Finally, the reader, who is the end consumer of all the published research, may greatly benefit by reading reports that clearly present what has been actually done during the conduct of the study. The reader may be a clinician seeking treatment ideas or treatment changes that may better serve his/her patients. The reader may be a student trying to further her knowledge on a subject or a health authority committee striving to draft treatment guidelines and healthcare policies. Clear and accurate reporting allows for better assessment and helps the readers to place the available evidence in the correct context and hierarchy level.

Have a look at the EQUATOR network site and see how the work of this group may help you improve your work. 